\title{
Erratum to: Cell-type-dependent access of HSF1 and HSF4 to $\alpha B$-crystallin promoter during heat shock
}

\author{
Zhe Jing • Rajendra K. Gangalum • Josh Z. Lee • \\ Dennis Mock • Suraj P. Bhat
}

Published online: 28 March 2013

(C) Cell Stress Society International 2013

\section{Erratum to: Cell Stress and Chaperones}

\section{DOI 10.1007/s12192-012-0386-7}

In the above mentioned publication, an incorrect version of Fig. 5 has been used.

Due to an unfortunate conversion error, the location of one of the red pentagons in Fig. 5b appeared incorrectly. The red pentagons representing enhanced HSF1 in heated $(H)$ NIH3T3 cells should be on the cryab gene promoter (as indicated below) and not on the schematics for the status of HSF4 on the cryab gene promoter (as incorrectly indicated in the original publication). Note that the originally published figure legend correctly describes the data as well as the schematics.

The correct image and its caption is published on the following page and should be treated as definitive by the reader.

The online version of the original article can be found at http:// dx.doi.org/10.1007/s12192-012-0386-7.

Z. Jing • R. K. Gangalum · J. Z. Lee · D. Mock · S. P. Bhat Jules Stein Eye Institute, David Geffen School of Medicine at UCLA, Brain Research Institute and Molecular Biology Institute, University of California, Los Angeles, CA 90095, USA

\section{S. P. Bhat $(\square)$}

Jules Stein Eye Institute, David Geffen School of Medicine at UCLA, University of California, Los Angeles, CA 90095, USA

e-mail: bhat@jsei.ucla.edu 
a

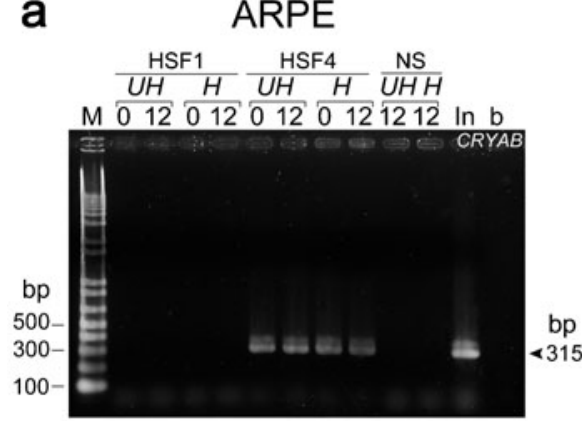

$\frac{\mathrm{HSF} 1}{U H \quad H} \frac{\mathrm{HSF}}{U H} \frac{\mathrm{HS}}{U H H}$

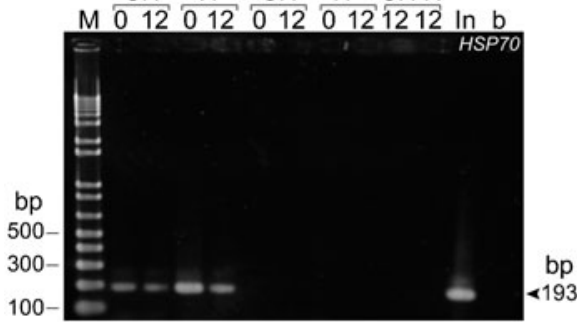

b

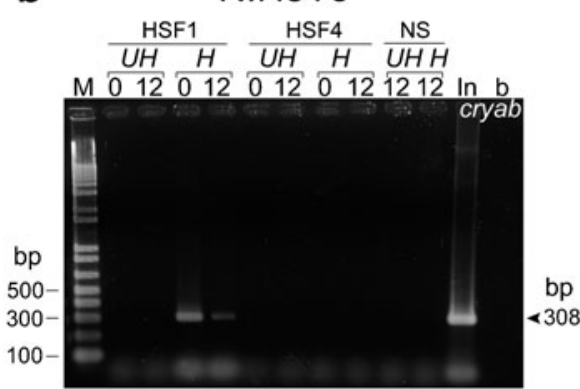
$\frac{\mathrm{HSF} 1}{\mathrm{UH} H} \frac{\mathrm{HSF} 4}{U H \quad H} \frac{\mathrm{NS}}{U H H}$ M $0120120120121212 \ln$ b
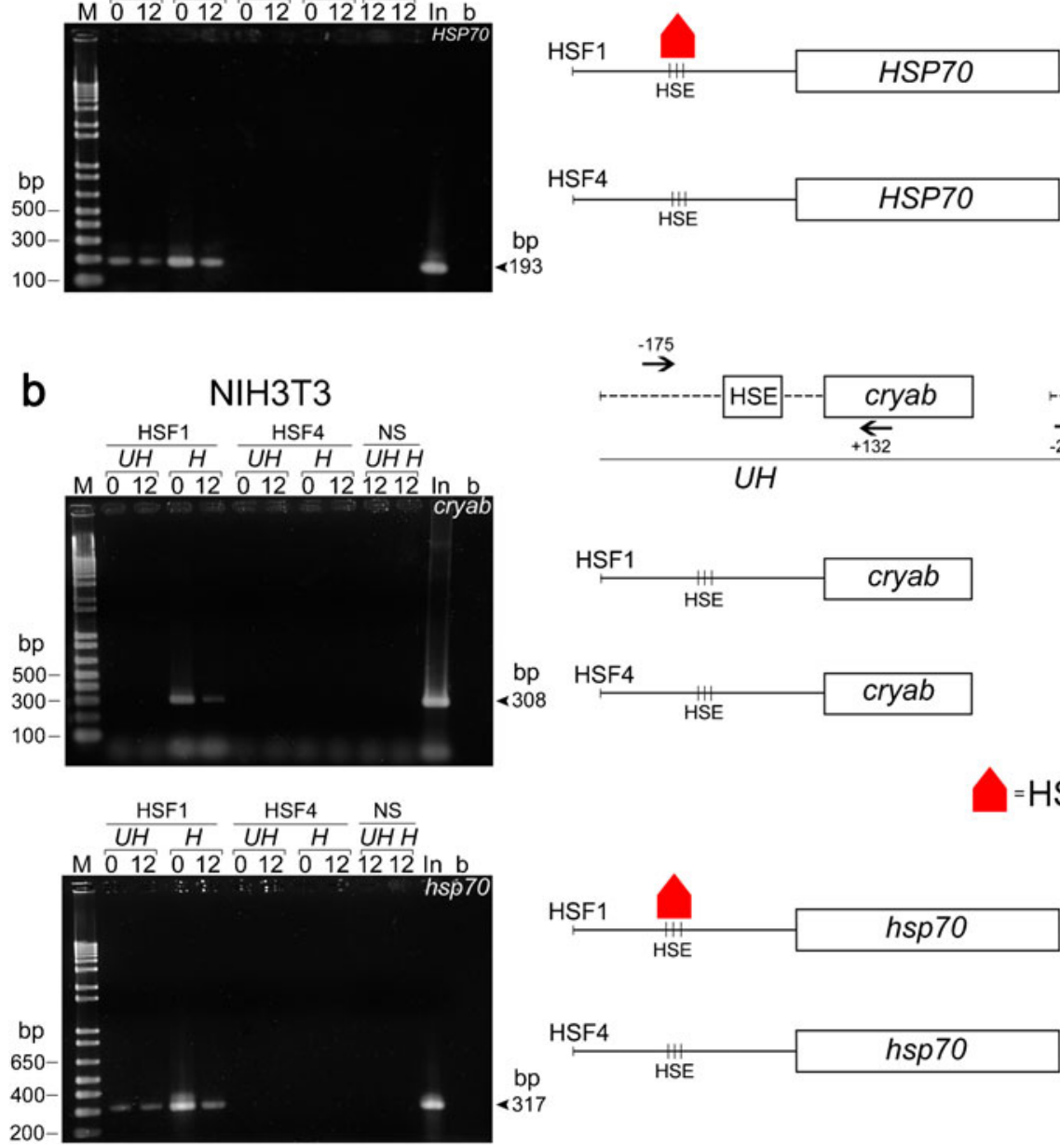

HSF4

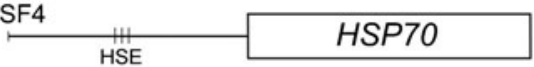

HSF4
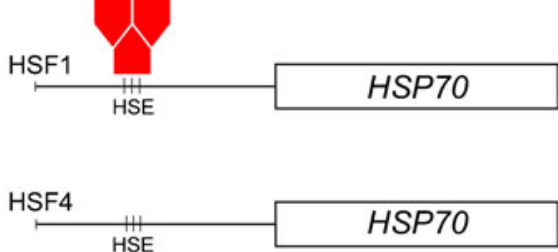

HSE
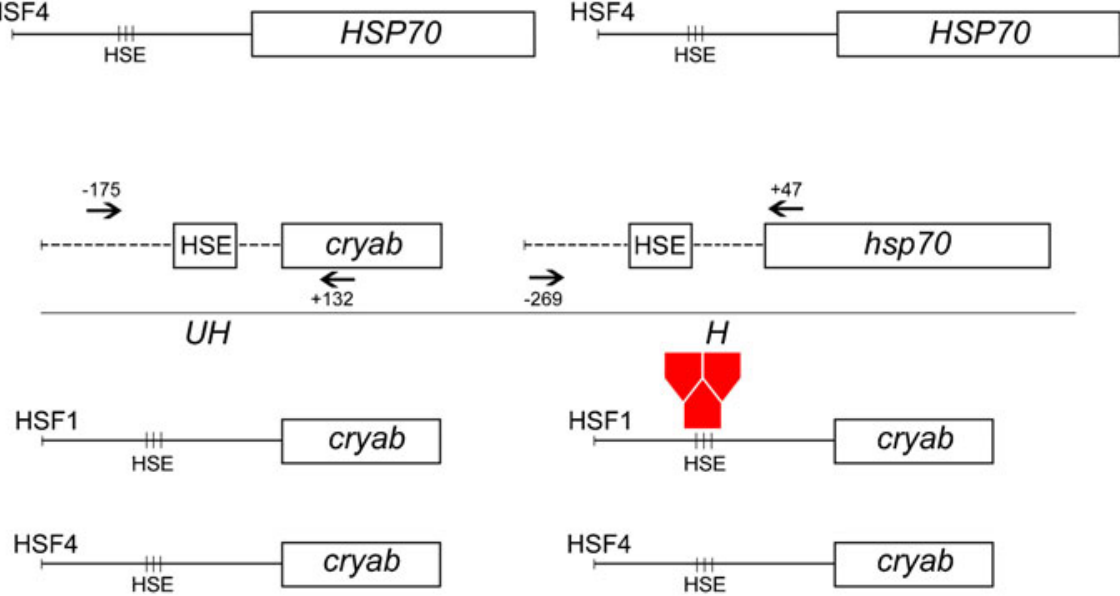

$=$ HSF 1
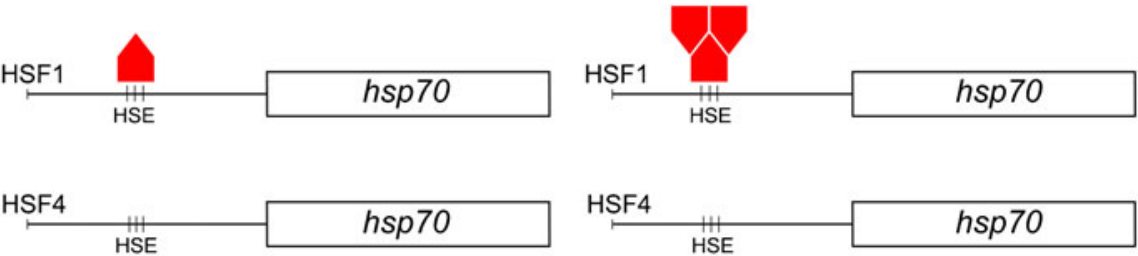

Fig. 5 Occupancy of $H S P 70$ and cryab promoters by HSF1 and HSF4 is cell-type specific. Native ARPE cells and NIH3T3 cells were processed for ChIP assay with HSF1 and HSF4-specific antibodies. The left panels show agarose gel electrophoresis of the PCR products. The right panels show schematic representation of the data obtained. a In ARPE cells there is no HSF1 on the cryab promoter either in control (unheated, $U H$ ) or heat shocked $(H)$ cells, either at $0 \mathrm{~h}$ or at $12 \mathrm{~h}$ postheat shock; HSF4 is seen only on the cryab promoter both in heated and unheated cells (blue ovals). There is no detectable HSF4 on the HSP70 promoter; HSF 1 is only seen on the HSP70 promoter in unheated cells, and its enhanced binding is seen in heat-shocked ARPE cells (red pentagons). b In NIH3T3 cells HSF1 is seen on the cryab promoter only in heated cells (red pentagons). HSF1 is also present on the $h s p 70$ promoter in both the heated as well as unheated cells (red pentagon), but it is enhanced in heat shocked cells (red pentagons) just as in ARPE cells in a. Note that there is no HSF4 on either the cryab promoter or the HSP70 promoter in NIH3T3 cells under any condition. The first lane of each gel shows the DNA markers ( $M, b p$ base pairs), the last lane (b) is the $\mathrm{H}_{2} \mathrm{O}$ control. $H$ heat shocked, $U H$ not heat shocked, In input DNA before immunoprecipitation, $N S$ normal serum. The sizes of amplicons are indicated on the right side of the agarose gels with an arrow pointing to the PCR product. The location of the primers for cryab and HSP70 promoters in ARPE and NIH3T3 cells are schematically depicted. This experiment was repeated twice 